\section{PLANNING IN THE TREATMENT OF CANCER}

BY

FRANK ELLIS, M.B., M.Sc., F.F.R., D.M.R.

Medical Director, Sheffield Radium Centre

Progress in medicine has mainly been achieved in the past, and will be furthered in the future, by the experimental approach to questions requiring solution. By this is not meant recourse to laboratories and experiments on animals only, important though the part may be which these factors play in elucidating our problems. Mackenzie (1918) stressed the importance of the experimental approach in clinical medicine, and it would seem that the cancer problem might gain considerably by clearsighted and logical planning of clinical experiments. The implications of the Cancer' Act make the planning of such experiments of great importance, and the present state of medical opinion renders them necessary.

\section{The Problem of Radiotherapy or Surgery}

Among certain sections of the profession engaged in the treatment of cancer there is a tendency to consider that an issue is at stake; that radiotherapy and surgery are alternatives advocated by their protagonists - an attitude which is tempered only by expediency on the one hand and necessity on the other. Only when an issue is a doubtful one does it provoke a conflict. When doubt is extinguished the conflict ceases. In the treatment of cancer this axiom applies, and the conflict will remain a reflection on the profession and a deterrent to progress unless a conscious effort is made to solve it.

To take one form of cancer considered by most people to-day to be a field for radiotherapy, the answers to certain questions are by no means clear. While the treatment of cancer of the cervix by radiotherapy is generally accepted as better than its treatment by surgery it is by no means proved that in certain selected cases surgical results are not more sure. Though radium treatment is known to result in a certain percentage of cases surviving for 5 years, and although supplementary $x$-ray treatment has been used for several years, it is not proved that the results of the combined treatment show any significant advance. Moreover, while some successes are to be expected from either radium or surgery alone, an inverse proportion of failures is also to be expected. The question thus presents itself: In what proportion of failures by radiotherapy would surgery have been successful, and vice versa?

Furthermore, the question of combined radiotherapy and surgery in this condition is of some importance from the point of view not only of continuing the individual patient's life but also in making that life as free from ill-health as possible. A uterus without cancer may still be better removed. No figures are at present available on this point, although Crossen and Newell (1935) showed that combined surgery and irradiation might be better than irradiation alone.

These problems in connexion with cancer of the cervix may be multiplied in connexion with cancer in any other situation. The aim is at present the best health attainable for every individual patient. It may never be possible to secure this, and with adequate progress in preventive methods and chemotherapy the problem may become archaic before it is solved. This being the common aim, however, for both surgeons and radiotherapists, they should combine to achieve it. The specialization which has become a feature of present-day surgery will provide an impetus to progress because difficulties requiring solution will be realized, methods become more uniform, and interest be more sustained.

\section{The Statistical Approach}

The peculiar feature of cancer treatment is that there are so many possible complicating factors that the result in any individual case can be accurately prophesied only in statistical terms. Similarly, the answer to any problem in connexion with cancer treatment must be sought on statistical lines. Suppose the problem is stated in its simplest terms. What Mr. Smith would like to know is whether he can obtain a better result by treating Mrs. Brown with radium or with surgery. Since he can treat the patient only once the problem is insoluble. The next best thing would be to treat with radium another patient, exactly like Mrs. Brown, having previously operated on Mrs. Brown, and then to observe the results. But it is impossible to keep them under exactly similar conditions for subsequent observation. The only way to solve the problem, therefore, is to treat so many patients with radium and so many with surgery that the differences to be expected between radium and surgical treatment of a single individual will be shown in terms of probability. Statistical methods are such that if large enough groups are chosen and only one factor is varied, an unequivocal statement of the relative effect of the varied factor can be made together with its probable error.

\section{Necessary Measures}

To answer the many questions that radiotherapists and surgeons can ask in connexion with cancer treatment, therefore, the following steps are necessary-having in view that the aim is to decide on the treatment in individual cases when the problems have been solved: (I) They must co-operate to decide on $a$ priori grounds and in the light of available evidence what are the problems requiring solution. (2) They must devise suitable experiments for each problem. (3) Each experiment must be carried out without variation in the conditions until a statistically certain result is achieved or the problem ceases to require solution because of developments on other lines, such as chemotherapy.

In an experiment it must be ensured that: $(a)$ no selection of cases occurs; (b) no change in methods. of treatment is permitted; $(c)$ no other factors are introduced; $(d)$ enough cases are treated to discover if there is a statistically significant difference in end-results; (e) cases are followed up and observed long enough to justify conclusions being drawn; $(f)$ adequate records for the purpose of the experiment are kept.

For instance, it may be decided to determine whether $x$ rays alone, contact $x$ rays after removal of the thyroid cartilage. or surgical removal by laryngofissure is the best method of treatment for intrinsic carcinoma of the larynx. The best way to avoid selection of cases is to ensure that for the purpose of the experiment only those which are suitable for treatment by any of the methods are included in the groups. Cases considered unsuitable, say, for laryngofissure would be excluded; but they could be included in another experiment -e.g., for determining the better of two radiotherapy techniques.

If the methods decided upon are then followed without variation and the results assessed statistically from time to time, an answer will certainly be obtained. The impending organization of radiotherapy into large centres together with regionalization of surgery will facilitate co-operation and ensure uniformity of method. This co-operation will destroy the antagonism between radiotherapists and surgeons and result in an alliance against cancer.

In this way plans can be made for dealing with all types of cancer so as eventually to know what must be done in any individual case to obtain the best result.

REFERENCES

Crossen, H. S., and Newell. Q. U. (1935). Amer. J. Obstet. Gynec., 29, 326 Mackenzie, Sir James (1920). Symptoms and their Interpretation, London,
pp. 1, 273.

The spring number (Volume VII, No. 1) of Art Notes (28a, Cornmarket Street, Oxford) is devoted as to 10 of its 16 pages to hospital planning. Mr. N. Notley, A.R.I.B.A., and Mr. R. Brown, in an article on this subject, suggest the principles that should be followed in choosing the site of a hospital, and in the general lay-out, the construction of wards and operating theatres, and the provision of heating and ventilation. Hospitals in Paris, Milan, New York, and Los Angeles are quoted as good examples, and Germany is considered to be in advance even of America in the planning of wards. The authors describe their design, submitted in a competition before the war, for the rebuilding of St. George's Hospital, but rot at Hyde. Park Corner. 